\title{
Implications of climate variability for the detection of multiple equilibria and for rapid transitions in the atmosphere-vegetation system
}

\author{
S. Bathiany $\cdot$ M. Claussen $\cdot$ K. Fraedrich
}

Received: 30 November 2010/ Accepted: 21 February 2011/Published online: 6 March 2011

(C) The Author(s) 2011. This article is published with open access at Springerlink.com

\begin{abstract}
Paleoclimatic records indicate a decline of vegetation cover in the Western Sahara at the end of the African Humid Period (about 5,500 years before present). Modelling studies have shown that this phenomenon may be interpreted as a critical transition that results from a bifurcation in the atmosphere-vegetation system. However, the stability properties of this system are closely linked to climate variability and depend on the climate model and the methods of analysis. By coupling the Planet Simulator (PlaSim), an atmosphere model of intermediate complexity, with the simple dynamic vegetation model VECODE, we assess previous methods for the detection of multiple equilibria, and demonstrate their limitations. In particular, a stability diagram can yield misleading results because of spatial interactions, and the system's steady state and its dependency on initial conditions are affected by atmospheric variability and nonlinearities. In addition, we analyse the implications of climate variability for the abruptness of a vegetation decline. We find that a vegetation collapse can happen at different locations at different times. These collapses are possible despite large and uncorrelated climate variability. Because of the nonlinear relation between vegetation dynamics and precipitation the green state is initially stabilised by the high variability. When precipitation falls below a critical threshold, the desert state is stabilised as variability is then also decreased.
\end{abstract}

S. Bathiany $(\bowtie) \cdot$ M. Claussen

Max Planck Institute for Meteorology, KlimaCampus,

Bundesstraße 53, 20146 Hamburg, Germany

e-mail: sebastian.bathiany@zmaw.de

M. Claussen · K. Fraedrich

Meteorologisches Institut, Universität Hamburg,

KlimaCampus, Bundesstraße 55, 20146 Hamburg, Germany
Keywords Green Sahara - Bifurcation - Multistability · Vegetation collapse . Tipping point .

Stochastic dynamical system

\section{Introduction}

Various proxy records have revealed that the Sahara was considerably greener and wetter during the early Holocene (Prentice et al. 2000). The reason lies in the earth orbit's precessional cycle, which caused an increased summer insolation and thus more precipitation. In particular, the West African Monsoon was enhanced due to an increased land-ocean temperature contrast. However, the extent of these changes can only be explained with positive feedbacks in the climate system (Braconnot et al. 2007). According to climate models the most important contribution is the atmosphere-vegetation feedback (Claussen 2009). First, the high albedo of bare soils in the Sahara with values up to 0.5 (Pinty et al. 2000) implies a low energy input to the overlying atmosphere. Therefore, the negative atmospheric radiative budget has to be partly compensated by diabatic warming caused by sinking motion. Convection and thus precipitation are then suppressed and vegetation cannot establish, a feedback proposed by Charney (1975) for the Sahel region. Second, vegetation can act to increase evapotranspiration at the expense of drainage and runoff, thus moistening the atmosphere and allowing for more precipitation (Hales et al. 2004). In addition, the contribution of the ocean-atmosphere feedback to the strength of the Holocene West African Monsoon is also supposed to be positive (Liu et al. 2003, 2004).

Climate modelling studies suggest that the atmospherevegetation feedback in Western Africa might allow for multiple equilibria of vegetation cover. This possibility can 
be illustrated with the conceptual model of Brovkin et al. (1998), which consists of two equilibrium curves $V^{*}(P)$ and $P^{*}(V)$, where $V$ stands for vegetation cover and $P$ for precipitation in a specific area (Fig. 1). While $V^{*}(P)$ is of nonlinear shape due to ecological thresholds, $P^{*}(V)$ is generally approximated as linear, because the impact of vegetation dynamics on precipitation is often small compared to geographical influences (Zeng et al. 2002). The intersections of the two curves determine the equilibrium points of the coupled system.

To detect multiple equilibria of vegetation cover, mainly two strategies have been applied so far: the choice of idealised initial conditions (such as complete forest and desert states), and the construction of a stability diagram similar to Fig. 1 with various methods. By choosing different initial conditions in ECHAM3-BIOME, Claussen (1994, 1997, 1998) have found two stable states for the Western Sahara for present day orbital forcing. Similar results have been obtained in models of intermediate complexity: Zeng and Neelin (2000) have identified multiple solutions for present day, though only "in a range of parameters at the margin of realistic estimates" (Zeng and Neelin, 2000). Wang and Eltahir (2000) have found even three stable states at present day, while Irizarry-Ortiz et al. (2003) have detected two stable states in the mid-Holocene. Multiple states in vegetation cover have even been obtained in tropical South America by Oyama and Nobre (2003) in an atmospheric general circulation model coupled to a potential vegetation model.

By using the conceptual model as a graphical method of stability analysis, Brovkin et al. (1998) have explained the existence of multiple equilibria in ECHAM3-BIOME by

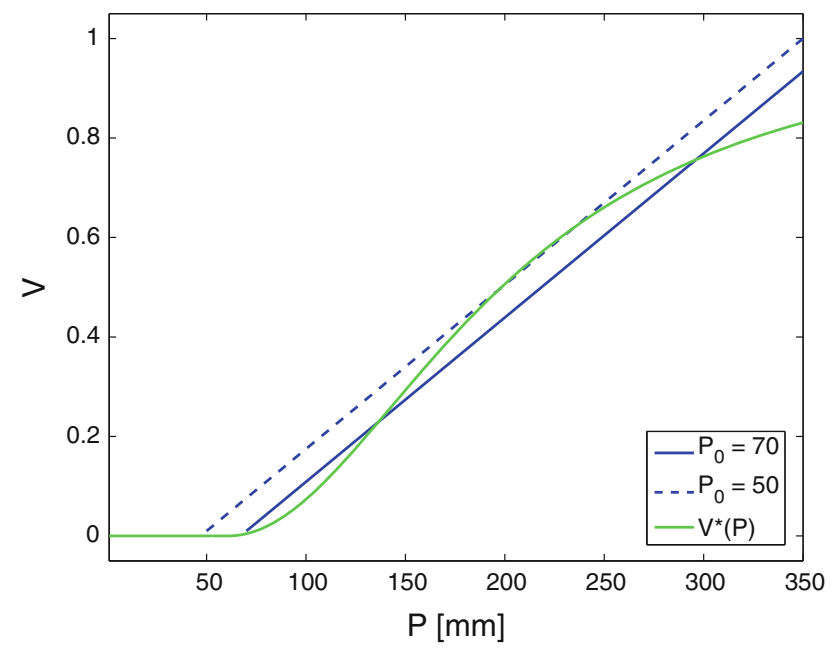

Fig. 1 Stability diagram after Brovkin et al. (1998) for $k=300 \mathrm{~mm}$. The blue lines represent the equilibrium of annual precipitation, calculated from $P^{*}(V)=P_{0}+k V$ for different $P_{0}$. The green line represents the equilibrium vegetation cover fraction $V^{*}(P)$ in VECODE for dry deserts (Eq. 1) with $G D D_{0}=10,000 \mathrm{~K}$ deriving parameters for the two equilibrium curves from the model output. For ECBilt-Clio-VECODE, a stability diagram also suggests the existence of multiple solutions (Renssen et al. 2003). Based on a similar approach Levis et al. (1999) and Brovkin et al. (2003) have concluded that no multiple equilibria are possible in boreal latitudes, despite a substantial positive feedback between near surface temperature and forest cover.

The possibility of multiple states in vegetation cover suggests the occurrence of sudden transitions between these states, which are either noise-induced (caused by an external disturbance), or the result of a bifurcation at a critical parameter value (tipping point). Indeed, there are proxy records showing a rapid decrease in West African vegetation at the end of the African Humid Period, some 1,000 years before present (e.g. deMenocal et al. 2000; Salzmann and Hoelzmann 2005). However, the timing and abruptness of this transition is site dependent. Other records indicate a more gradual transition in more easterly locations (Kroepelin et al. 2008; Lezine 2009), and in tropical Africa (Vincens et al. 2010).

The abruptness of this decline in vegetation cover also differs among climate models. While Claussen et al. (1999) have obtained a transition that is much more rapid than the change in orbital forcing, Renssen et al. $(2003,2006)$ as well as Schurgers et al. (2006) have only obtained a gradual vegetation decline. Furthermore, multistability does not necessarily imply an abrupt transition, or vice versa. The reason lies in the versatile effects of climate variability. Variability can smooth the gradients in vegetation cover and even lead to steady states that are distinct from any deterministic equilibrium (Zeng and Neelin 2000; Zeng et al. 2002; D'Odorico et al. 2005). Furthermore, variability can even lead to the establishment of multiple stable states, as has been demonstrated with a coupled climate-ecosystem model by Liu (2010), and with a box model for the North Atlantic Meridional Overturning Circulation by Timmermann and Lohmann (2000). If it is sufficiently high, variability can obliterate a system's dependency on initial conditions (Wang 2004) and remove hysteresis effects (Guttal and Jayaprakash 2007). In turn, variability itself can be influenced by the stability properties. For example, a flickering between two regimes can cause low-frequency variations (Wang and Eltahir 2000; Wang 2004) and a bimodal probability distribution (Livina et al. 2010). The transition of a system passing a deterministic tipping point may then be only gradual, in the sense that the state's probability density changes only gradually when the critical parameter is varied. In contrast, rapid shifts are also possible in monostable systems: To explain the rapid vegetation decline in the complex atmosphere-ocean-land model FOAM-LPJ, Liu et al. (2006) have suggested low-frequency variations in precipitation, independent of vegetation 
dynamics. Due to the vegetation's nonlinear dependency on soil moisture in arid regions, a rapid vegetation decline is then possible even in case of an only weak atmospherevegetation feedback.

In this study we further investigate the implications of climate variability for the detection of multiple equilibria and the possibility of rapid transitions. In Sect. 2 we describe the models and our experiment setups. We then present transient simulations of the mid-Holocene (Sect. 3), and apply and assess the methods of stability diagram analysis (Sect. 4.1) and extreme initial conditions (Sect. 4.2) We explain our results with a simple stochastic model, and in Sect. 5 apply it to document a new mechanism of vegetation collapse. Section 6 provides a short summary, a discussion of the implications for reality and other models, and our basic conclusions.

\section{Model and experiment setup}

\subsection{Atmosphere model}

The Planet Simulator (PlaSim; Fraedrich et al. 2005a, b) is a global spectral climate model of intermediate complexity, which is freely available as an open source code (http:// www.mi.uni-hamburg.de/Planet-Simul.216.0.html). We run PlaSim with T21 horizontal resolution and 10 vertical layers. As we only consider biogeophysical land-atmosphere feedbacks, atmospheric $\mathrm{CO}_{2}$ is set to $280 \mathrm{ppm}$ in all experiments, and ocean surface temperatures and sea ice properties are prescribed from present day observations and are the same in each year.

\subsection{Vegetation model}

Vegetation dynamics are represented by VECODE (Brovkin et al. 2002), a dynamic global vegetation model of reduced complexity. With a timestep of 1 year, the equilibrium cover fractions of trees, grass and desert are calculated from precipitation, growing degree days above $0^{\circ} \mathrm{C}$ $\left(G D D_{0}\right)$, and temperature. For this transformation analytical functions are used whose parameters have been tuned in order to match the observed relation between vegetation distribution and climate (Brovkin et al. 1997). For dry deserts, the equilibrium vegetation fraction $V^{*}$ is calculated from annual precipitation $P$ via

$V^{*}=1-d^{*}$

with

$$
d^{*}=\left\{\begin{array}{cl}
1 & \text { if } P<P_{1} \\
0 & \text { if } P>P_{2} \\
\frac{1.03}{1+\alpha\left(\frac{P-\beta \exp (\gamma \delta / 2)}{\exp (\gamma \delta)}\right)^{2}}-0.03 & \text { else, }
\end{array}\right.
$$

where

$P_{1}=\beta \exp (\gamma \delta / 2)$

$P_{2}=\beta \exp (\gamma \delta / 2)+\frac{\exp (\gamma \delta)}{\sqrt{0.03 \alpha}}$.

Parameter values are $\alpha=0.0011, \beta=28, \gamma=1.7 \times$ $10^{-4}$, and $\delta=G D D_{0}-900 \mathrm{~K}$. The second condition corresponds to resetting $d^{*}$ to 0 once it becomes negative.

For exploring abrupt vegetation dynamics, we create a second, more sensitive model by setting $\beta=140$ and $\gamma=1.7 \times 10^{-5}$ (Fig. 2, dashed line). By doing so we accept that the modified VECODE is less realistic than the original one. As the strength of the atmosphere-vegetation feedback and the existence of multiple steady states in reality remains unclear (Liu et al. 2007; Claussen 2009), we aim to also represent a case of a particularly large feedback. To do this, we prefer the mentioned approach because the sensitivity of VECODE can be changed much easier and more directly than the sensitivity of the atmosphere model.

\subsection{Coupling procedure}

VECODE is coupled to PlaSim in two ways, equilibrium mode and transient mode. In equilibrium mode we run the atmosphere model for 10 years (if not stated otherwise), and average the climate over this period. The corresponding equilibrium cover fractions then provide the boundary conditions in PlaSim for the next iteration step. This approach is similar to previous studies like Claussen (1994) and Liu et al. (1999). In transient mode, the cover fractions are updated each year and approach their (annually

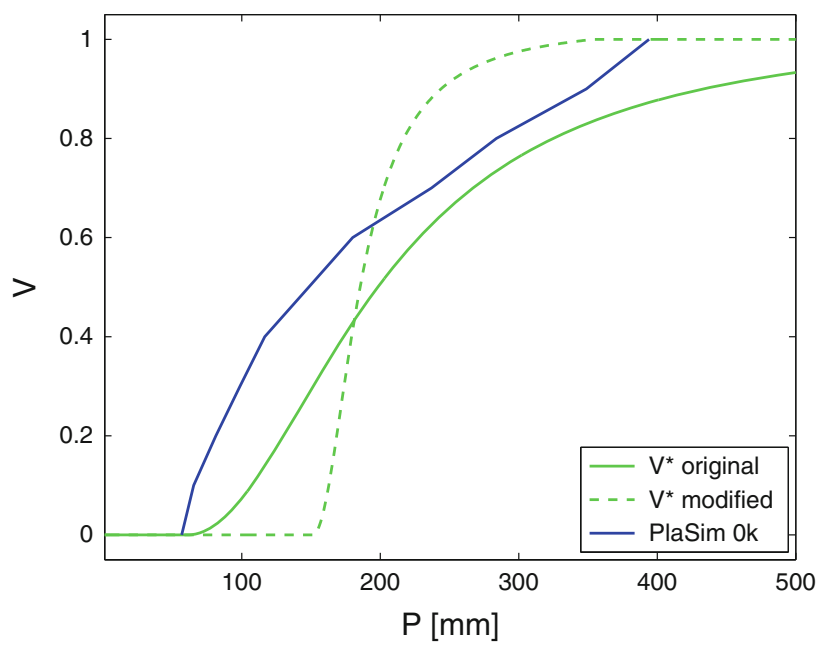

Fig. 2 Equilibrium vegetation cover fraction $V^{*}(P)$ for both VECODE versions. The blue line shows the spatial mean $P^{*}(V)$ response of uncoupled PlaSim simulations with fixed uniform grass cover in the Sahara/Sahel region and present day orbital forcing 
changing) equilibrium values on the basis of an exponential filter. A linearisation leads to a relaxation law commonly used in simple dynamic vegetation models (e.g. Zeng and Neelin 2000; Zeng et al. 2002; Wang 2004; Liu et al. 2006):

$\frac{d V}{d t}=\frac{V^{*}-V}{\tau}$.

A similar equation is solved for tree cover. The cover fraction of grass is then determined as the difference between vegetation and tree cover, so that there is coexistence but no competition between trees and grass. In the dry regions of the Sahara/Sahel we are interested in, vegetation almost completely consists of grass so that vegetation dynamics can be described by Eq. 2 with $\tau$ as the timescale of grass. In VECODE, $\tau$ is calculated from net primary production (NPP), which depends on annual precipitation, annual mean temperature and atmospheric $\mathrm{CO}_{2}$. These dependencies are implemented as least squares fits of analytical curves to global observations of NPP, living phytomass, and climate. Interannual changes in $\tau$ are mostly due to the variability in precipitation: In wet years, vegetation dynamics are faster than in dry years (Fig. 3).

In each coupling step, the land cover types have to be transformed to surface parameter values. In PlaSim, the four parameters that are substantially affected by vegetation are background (snowfree) surface albedo, surface roughness length, bucket size and a parameter that controls the fraction of soil water available for evaporation (Fraedrich et al. 2005a, b). For each land cover type (trees, grass and desert) we assume constant parameter values (Table 1) that are combined by weighting with the land cover fractions in a particular grid cell. Most parameter values for trees and desert are from Fraedrich et al. (2005a,

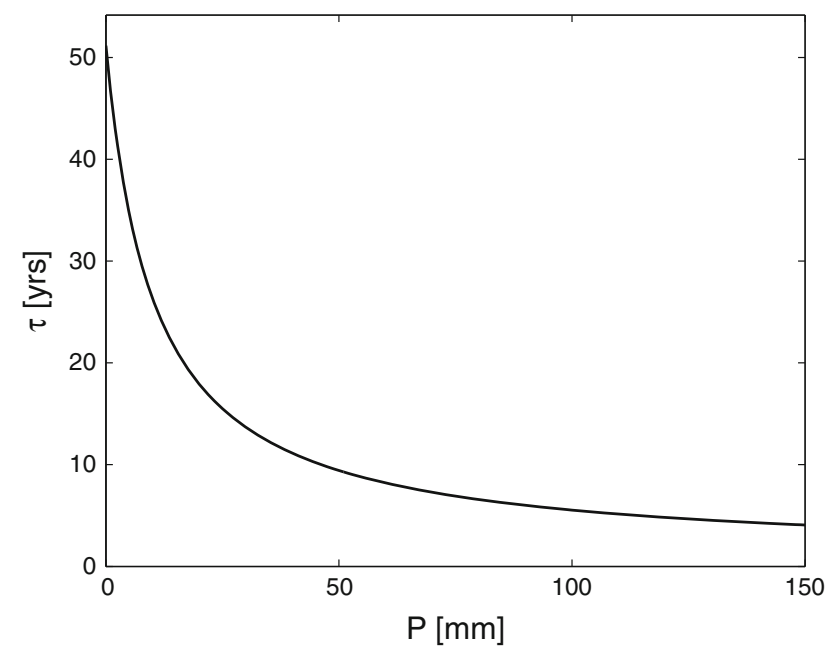

Fig. 3 Timescale for shifts in grass fraction in dependence on annual precipitation, at a temperature of $27^{\circ} \mathrm{C}$, and $280 \mathrm{ppm} \mathrm{CO}_{2}$ in VECODE
Table 1 Attribution of surface parameter values to VECODE land cover types

\begin{tabular}{lllll}
\hline & $\begin{array}{l}\text { Background } \\
\text { albedo }\end{array}$ & $\begin{array}{l}\text { Roughness } \\
\text { length }[\mathrm{m}]\end{array}$ & $\begin{array}{l}\text { Bucket } \\
\text { size }[\mathrm{m}]\end{array}$ & $\begin{array}{l}\text { Fractional } \\
\text { parameter }\end{array}$ \\
\hline Trees & $0.12^{\mathrm{a}}$ & $1.5^{\mathrm{b}}$ & $0.5^{\mathrm{a}}$ & $0.01^{\mathrm{a}}$ \\
Grass & $0.16^{\mathrm{b}}$ & $0.05^{\mathrm{b}}$ & 0.2 & 0.1 \\
Desert & $0.28^{\mathrm{a}} / 0.4$ & $0.05^{\mathrm{b}}$ & $0.1^{\mathrm{a}}$ & $0.4^{\mathrm{a}}$ \\
\hline
\end{tabular}

${ }^{a}$ Value from Fraedrich et al. (2005a, b)

b Value from Claussen (1994)

b), in the other cases we chose values in agreement to the typical biomes in Claussen (1994). In the case of roughness length we average the corresponding momentum transfer coefficients for neutral atmospheric stability at a height of $1,000 \mathrm{~m}$ (Stull 1988). In addition, this bulk vegetation roughness length is combined with orographic roughness by taking the root of their summed squares (Claussen 1994). Surface albedo, bucket size and the fractional evaporation parameter are averaged linearly. For soil albedo Fraedrich et al. (2005a, b) chose a value of 0.28 globally, which is the maximum surface albedo at snowand icefree land cells in the uncoupled PlaSim. However, as the large differences in surface albedo between bare and vegetated ground in the Sahara/Sahel region are required to capture the local vegetation-atmosphere feedback, we modify the desert background albedo in Northern Africa and Arabia (approx. $14^{\circ} \mathrm{W}-47^{\circ} \mathrm{E}, 12-32^{\circ} \mathrm{N}$ ). There, we assume a value of 0.4 , while for the rest of the world we chose 0.28 .

\subsection{Experiments}

We perform different types of experiments (Table 2), whose details will be explained together with the results in the following sections:

- Experiments in transient mode with continuously changing orbital parameters, corresponding to the period from 8,000 years before present $(8 \mathrm{k})$ to 2,000 years before present $(2 \mathrm{k})$

- Experiments starting from idealised initial conditions (forest and desert world) with fixed orbital forcing, for both ways of coupling and both VECODE versions

- Uncoupled PlaSim experiments of several 1,000 years length, with fixed orbit year, and surface parameters fixed to the mean conditions obtained from the transiently coupled experiments (after a steady state was reached)

- Experiments with a simple stochastic model that is based on Eqs. 1 and 2, but describes atmospheric variability as a random process $\eta$ with noise level $\sigma_{P}$. In analogy to the stability diagram of Brovkin et al. 
Table 2 List of experiments with PlaSim-VECODE

\begin{tabular}{|c|c|c|c|c|c|}
\hline Experiment & Coupling mode and frequency & VECODE version & Orbit year & Initial surface conditions & Multiple states \\
\hline T-Orig-for1 & $\operatorname{Tr}$ & Orig & $8 \mathrm{k}-2 \mathrm{k}$ & Default & \\
\hline T-Orig-for2 & $\operatorname{Tr}$ & Orig & $8 \mathrm{k}-2 \mathrm{k}$ & Default & \\
\hline T-Mod-for1 & $\operatorname{Tr}$ & Mod & $8 \mathrm{k}-2 \mathrm{k}$ & Default & \\
\hline T-Mod-for2 & $\operatorname{Tr}$ & Mod & $8 \mathrm{k}-2 \mathrm{k}$ & Default & \\
\hline T-Orig-back1 & $\operatorname{Tr}$ & Orig & $2 \mathrm{k}-8 \mathrm{k}$ & Default & \\
\hline T-Orig-back2 & $\operatorname{Tr}$ & Orig & $2 \mathrm{k}-8 \mathrm{k}$ & Default & \\
\hline T-Mod-back1 & $\operatorname{Tr}$ & Mod & $2 \mathrm{k}-8 \mathrm{k}$ & Default & \\
\hline T-Mod-back2 & $\operatorname{Tr}$ & Mod & $2 \mathrm{k}-8 \mathrm{k}$ & Default & \\
\hline E1F-Mod-8k & Eq 1 year & Mod & $8 \mathrm{k}$ & Forest world & No \\
\hline E1D-Mod-8k & Eq 1 year & Mod & $8 \mathrm{k}$ & Desert world & \\
\hline E3F-Mod-8k & Eq 3 years & Mod & $8 \mathrm{k}$ & Forest world & No \\
\hline E3D-Mod-8k & Eq 3 years & Mod & $8 \mathrm{k}$ & Desert world & \\
\hline E5F-Mod-8k & Eq 5 years & Mod & $8 \mathrm{k}$ & Forest world & Yes \\
\hline E5D-Mod-8k & Eq 5 years & Mod & $8 \mathrm{k}$ & Desert world & \\
\hline E7F-Mod-8k & Eq 7 years & Mod & $8 \mathrm{k}$ & Forest world & Yes \\
\hline E7D-Mod-8k & Eq 7 years & Mod & $8 \mathrm{k}$ & Desert world & \\
\hline E10F-Mod-8k & Eq 10 years & Mod & $8 \mathrm{k}$ & Forest world & Yes \\
\hline E10D-Mod-8k & Eq 10 years & Mod & $8 \mathrm{k}$ & Desert world & \\
\hline E20F-Mod-7k & Eq 20 years & Mod & $7 \mathrm{k}$ & Forest world & Yes \\
\hline E20D-Mod-7k & Eq 20 years & Mod & $7 \mathrm{k}$ & Desert world & \\
\hline E10F-Mod-7k & Eq 10 years & Mod & $7 \mathrm{k}$ & Forest world & No \\
\hline E10D-Mod-7k & Eq 10 years & Mod & $7 \mathrm{k}$ & Desert world & \\
\hline E10F-Mod-6k & Eq 10 years & Mod & $6 \mathrm{k}$ & Forest world & No \\
\hline E10D-Mod-6k & Eq 10 years & Mod & $6 \mathrm{k}$ & Desert world & \\
\hline E10F-Mod-5.5k & Eq 10 years & Mod & $5.5 \mathrm{k}$ & Forest world & No \\
\hline E10D-Mod-5.5k & Eq 10 years & Mod & $5.5 \mathrm{k}$ & Desert world & \\
\hline E10F-Mod-5k & Eq 10 years & Mod & $5 \mathrm{k}$ & Forest world & Yes \\
\hline E10D-Mod-5k & Eq 10 years & Mod & $5 \mathrm{k}$ & Desert world & \\
\hline E10F-Mod-4.5k & Eq 10 years & Mod & $4.5 \mathrm{k}$ & Forest world & Yes \\
\hline E10D-Mod-4.5k & Eq 10 years & Mod & $4.5 \mathrm{k}$ & Desert world & \\
\hline E10F-Mod-0k & Eq 10 years & Mod & $0 \mathrm{k}$ & Forest world & No \\
\hline E10D-Mod-0k & Eq 10 years & Mod & $0 \mathrm{k}$ & Desert world & \\
\hline TF-Mod-8k & $\operatorname{Tr}$ & Mod & $8 \mathrm{k}$ & Forest world & No \\
\hline TD-Mod-8k & $\operatorname{Tr}$ & Mod & $8 \mathrm{k}$ & Desert world & \\
\hline TF-Mod-4.5k & $\operatorname{Tr}$ & Mod & $4.5 \mathrm{k}$ & Forest world & No \\
\hline TD-Mod-4.5k & $\operatorname{Tr}$ & Mod & $4.5 \mathrm{k}$ & Desert world & \\
\hline TF-Mod-0k & $\operatorname{Tr}$ & Mod & 0k & Forest world & No \\
\hline TD-Mod-0k & $\operatorname{Tr}$ & Mod & 0k & Desert world & \\
\hline E10F-Orig-8k & Eq 10 years & Orig & $8 \mathrm{k}$ & Forest world & No \\
\hline E10D-Orig-8k & Eq 10 years & Orig & $8 \mathrm{k}$ & Desert world & \\
\hline E10F-Orig-6k & Eq 10 years & Orig & $6 \mathrm{k}$ & Forest world & No \\
\hline E10D-Orig-6k & Eq 10 years & Orig & $6 \mathrm{k}$ & Desert world & \\
\hline E10F-Orig-5.5k & Eq 10 years & Orig & $5.5 \mathrm{k}$ & Forest world & No \\
\hline E10D-Orig-5.5k & Eq 10 years & Orig & $5.5 \mathrm{k}$ & Desert world & \\
\hline E10F-Orig-5k & Eq 10 years & Orig & $5 \mathrm{k}$ & Forest world & No \\
\hline E10D-Orig-5k & Eq 10 years & Orig & $5 \mathrm{k}$ & Desert world & \\
\hline E10F-Orig-4.5k & Eq 10 years & Orig & $4.5 \mathrm{k}$ & Forest world & No \\
\hline E10D-Orig-4.5k & Eq 10 years & Orig & $4.5 \mathrm{k}$ & Desert world & \\
\hline
\end{tabular}


Table 2 continued

\begin{tabular}{lllll}
\hline Experiment & Coupling mode and frequency & VECODE version & Orbit year & Initial surface conditions \\
\hline E10F-Orig-0k & Eq 10 years & Orig & $0 \mathrm{k}$ & Forest world \\
E10D-Orig-0k & Eq 10 years & Orig & $0 \mathrm{k}$ & Desert world \\
T-Mod-8k & $\operatorname{Tr}$ & Mod & $8 \mathrm{k}$ & Default \\
T-Mod-7k & $\mathrm{Tr}$ & Mod & $7 \mathrm{k}$ & Default \\
T-Mod-4.5k & $\mathrm{Tr}$ & Mod & $4.5 \mathrm{k}$ & Default \\
T-Mod-4k & $\operatorname{Tr}$ & Mod & $4 \mathrm{k}$ & Default \\
T-Mod-3.6k & $\operatorname{Tr}$ & Mod & $3.6 \mathrm{k}$ & Default \\
T-Orig-8k & $\operatorname{Tr}$ & Orig & $8 \mathrm{k}$ & Default \\
TU-8k & Uncoupled & - & $8 \mathrm{k}$ & Years 300-1,000 from T-Orig-8k \\
T-Orig-5.5k & $\operatorname{Tr}$ & Orig & $5.5 \mathrm{k}$ & Default \\
TU-5.5k & Uncoupled & - & $5.5 \mathrm{k}$ & Years 300-1,000 from T-Orig-5.5k \\
T-Orig-0k & $\operatorname{Tr}$ & Orig & $0 \mathrm{k}$ & Default \\
TU-0k & Uncoupled & - & $0 \mathrm{k}$ & Years 300-1,000 from T-Orig-0k \\
\hline
\end{tabular}

In case of the uncoupled PlaSim experiments the initial surface conditions provide the boundary conditions during the complete experiment. "Default" refers to the original surface parameters in PlaSim. The existence of multiple states was determined by eye from the according time series, like those in Fig. 7

$\operatorname{Tr}$ transient coupling mode, Eq equilibrium coupling mode

(1998) we assume a linear relation between equilibrium precipitation and vegetation cover fraction:

$P=P_{0}+k V+\sigma_{P} \eta$.

In this regard our stochastic model is essentially the same as in Wang (2004) and Liu et al. (2006).

\section{Vegetation dynamics from $8 k$ to $2 k$}

We run PlaSim-VECODE in transient mode and with both VECODE versions under changing orbital forcing from $8 \mathrm{k}$ to $2 \mathrm{k}$. Each experiment is conducted twice with the same settings but different initial conditions in order to get an impression on the stochasticity of the time series. These alternative initial conditions are created by keeping the initial vegetation cover fixed for 2 years instead of one. In the following, we refer to the $9 \times 4$ grid cells in the area $14^{\circ} \mathrm{W}-36^{\circ} \mathrm{E}, 12-32^{\circ} \mathrm{N}$ as the Sahara/Sahel region. In this section we will use only descriptive terms like vegetation decline regardless of the underlying mechanism, while we reserve the term collapse for transitions that are related to a bifurcation.

In all transient experiments, mean vegetation cover in the Sahara/Sahel remains almost stationary during the first 2,000 years, whereas a comparatively rapid decrease occurs between $6 \mathrm{k}$ and $5 \mathrm{k}$ (Fig. 4). For the original VECODE, large fluctuations occur in the 100-year moving averages during the transition period, until the rate of vegetation decline decreases again around $5 \mathrm{k}$. For the modified VECODE, this vegetation decrease is more abrupt and occurs in one major event. The evolution of spatial mean precipitation in the Sahara/Sahel region closely resembles the vegetation dynamics, although absolute changes are small between $5 \mathrm{k}$ and $2 \mathrm{k}$.

An analysis of the local changes reveals that the rapid transition also occurs in Central Arabia and the Middle East. For the modified VECODE, a second, even more pronounced vegetation decline occurs between $4 \mathrm{k}$ and $3 \mathrm{k}$ in the south-western Sahara (Fig. 5). While these features can be seen in all experiments, the shape of each time series and the exact timing of the transitions differ among the two ensemble members.

\section{Detection of multiple equilibria}

\subsection{On the use of stability diagrams}

Stability diagrams like Fig. 1 illustrate the nature of multistability, but they have also been used to infer the equilibria for a particular region in a complex climate model (Brovkin et al. 2003; Wang 2004). When simulating the Holocene with ECBilt-CLIO-VECODE, Renssen et al. $(2003,2006)$ obtained a rather gradual decline in vegetation cover in the Sahara/Sahel region, even more gradual than our result with the original VECODE, considering the smaller region in their Fig. 1c (Renssen et al. 2003). However, the construction of a stability diagram lead to the conclusion that multiple equilibria exist in the early to midHolocene in ECBilt-CLIO-VECODE. The gradual decline 
Fig. 4 Evolution of spatial mean vegetation cover fraction (left) and precipitation in $\mathrm{mm} /$ year (right) in the Sahara/Sahel region in two PlaSim-VECODE experiments with the original (four top panels) and modified VECODE (four bottom panels). Thin line: annual data; heavy line: 100-year running mean. Time is in 1,000 years before present
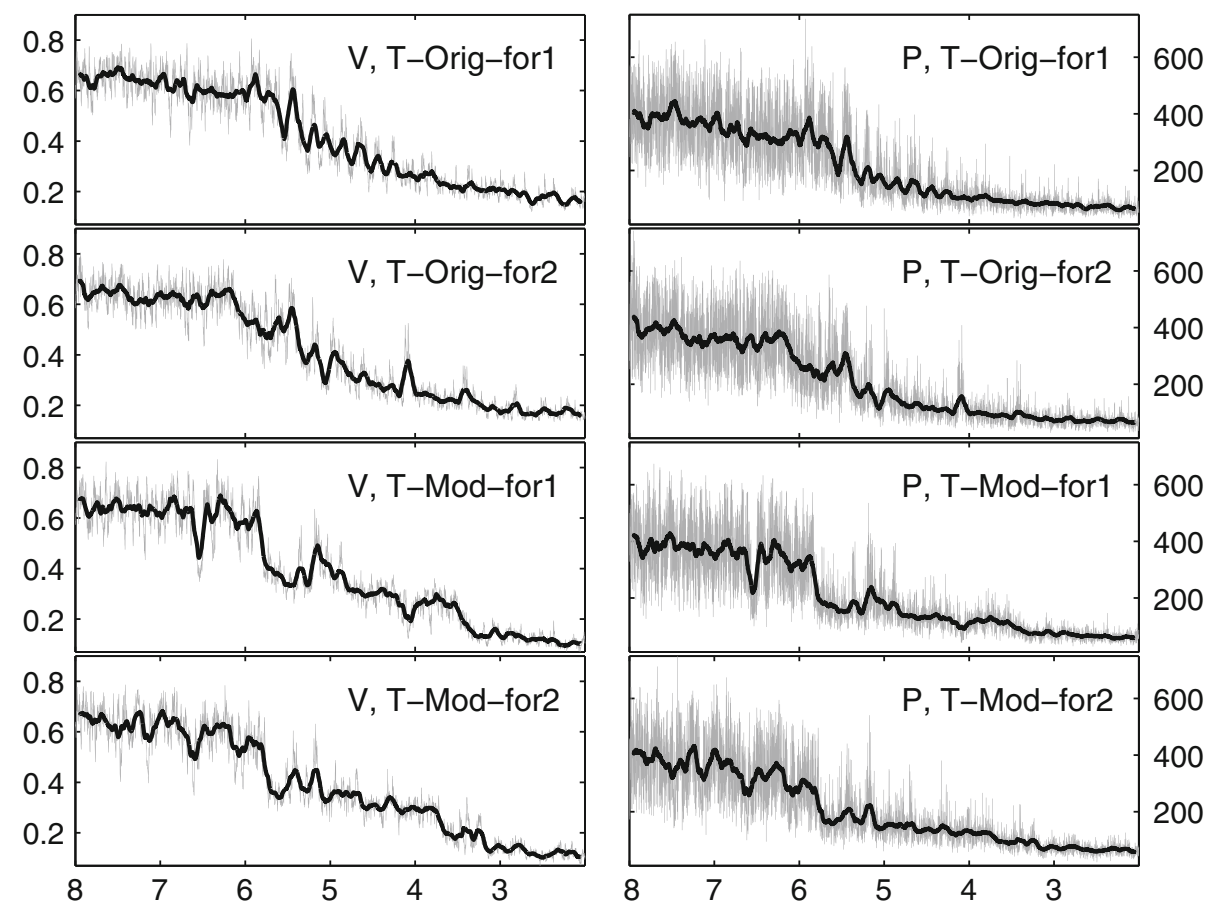
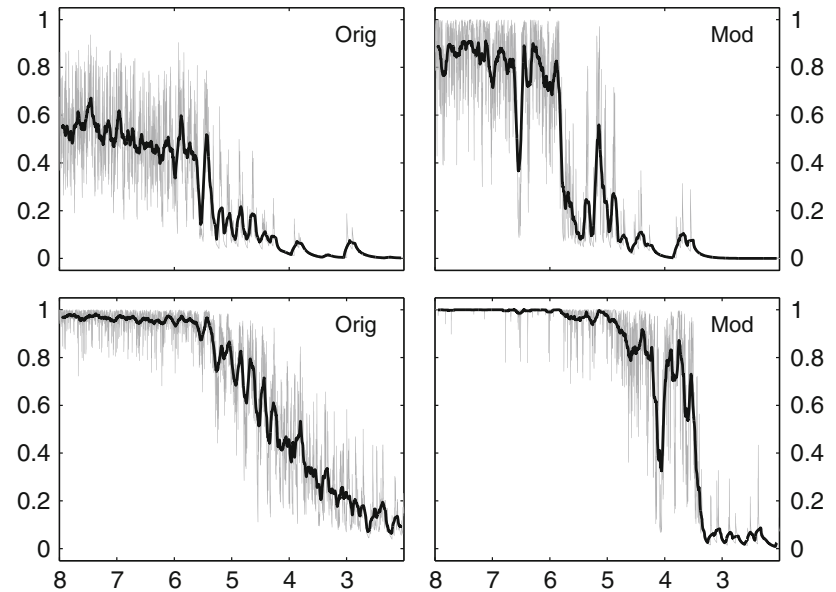

Fig. 5 Evolution of vegetation cover fraction at two adjacent grid cells in the western Sahara (approx. $0^{\circ}, 12-22^{\circ} \mathrm{N}$; Fig. 6), for the transient experiments T-Orig-for1 (left panels) and T-Mod-for1 (right panels). Thin line: annual data; heavy line: 100-year running mean. Time is in 1,000 years before present

in vegetation cover was explained with the large atmospheric variability that supposedly caused frequent shifts between the equilibrium states.

The specific method to construct a stability diagram differs among publications. Renssen et al. (2003) derived the $P^{*}(V)$-line by connecting two points in phase space: the mean state of a coupled experiment and the mean state of an uncoupled experiment with fixed vegetation cover. When doing so the following limitations have to be considered: (1) The mean state of the coupled model does not necessarily correspond to a deterministic equilibrium. (2)
The equilibrium curve $P^{*}(V)$ is not strictly linear. (3) Growing degree days are not a constant but differ from grid cell to grid cell, and also depend on vegetation cover.

However, we argue that the main caveat is the high dimensionality that cannot be adequately represented in a simple one-dimensional model. To demonstrate this we construct a stability diagram for $0 \mathrm{k}$ conditions with the following method:

- In 11 different experiments we prescribe a uniform and fixed grass cover in the Sahara/Sahel region, with cover fractions ranging from 0 to 1 in steps of 0.1 . Each of these uncoupled PlaSim simulations has a length of 100 years, of which the last 90 years are averaged over time and over the Sahara/Sahel region.

- The resulting mean annual precipitation values of the 11 experiments represent the equilibrium precipitation curve $P^{*}(V)$ in the stability diagram.

- We draw the $V^{*}(P)$ curve (Eq. 2) by assuming a fixed $G D D_{0}$ value of $10,000 \mathrm{~K}$. An analysis which takes into account that $G D D_{0}$ differs among grid cells and among the experiments leads to a different vegetation curve, but similar results regarding the number of intersections between $V^{*}$ and $P^{*}$.

The resulting stability diagram (Fig. 2) clearly diagnoses two stable solutions for the modified VECODE that may also exist for the original version at an earlier time than 0k. However, we will show in Sect. 4.2 that no multiple equilibria exist in $0 \mathrm{k}$. The reason for this contradiction lies in the spatial heterogeneity and the interactions among 
all grid cells via the atmosphere. For example, when Renssen et al. (2003) fixed vegetation cover to 0 in their region of analysis, the resulting mean precipitation could not sustain vegetation if it occurred at a single grid cell. Therefore, the one-dimensional stability diagram indicated a desert equilibrium. In the spatially resolved model some grid cells can nonetheless maintain vegetation which in turn potentially increases precipitation also at other cells. The alleged desert equilibrium may thus be nonexistent, because the one-dimensional simplification is not justified. We therefore argue that the construction of a stability diagram may yield misleading conclusions and in the following focus on the method of different initial conditions.

\subsection{Extreme initial conditions: desert and forest world}

\subsubsection{PlaSim-VECODE results}

In order to interpret the results of the transient experiments in terms of multiple equilibria, we run the coupled PlaSimVECODE model starting from different initial conditions. This strategy may generally not be a trivial task, as it is not always clear how to chose the initial conditions. Also, more than two possible solutions could exist (e.g. Wang and Eltahir 2000; Dekker et al. 2010). Putting these restrictions aside, we chose the extreme initial conditions of global desert and global forest (Fraedrich et al. 1999, 2005a, b). In both cases, all land points except ice shields are initialised with the surface parameter values corresponding to complete forest or desert, respectively. To bring the free variables to an equilibrium with the extreme surface parameters, we run PlaSim for 15 years under forest and desert world conditions. After this spinup time the land cover types are allowed to change. We use this initialisation procedure regardless of the coupling method or VECODE version, and run the model until a steady state is obtained. The results are summarized as follows:

- For the original VECODE we find no multiple steady states. All model trajectories lead to the same mean state. This result is independent of time (between $8 \mathrm{k}$ and 0k), the way of coupling (transient or equilibrium mode) and the coupling frequency in equilibrium mode (up to 20 years).

- For the modified VECODE in transient mode, we find no multiple steady states, independently of the orbit year $(8 \mathrm{k}, 4.5 \mathrm{k}$, and $0 \mathrm{k})$.

- For the modified VECODE in equilibrium mode, we find multiple steady states, whose extent and location depends on the orbit year (Fig. 6). In 8k the bistable region extends from the southern margin of the Sahara desert to the central Arabian peninsula and the Middle East. In $4.5 \mathrm{k}$, the only substantial difference between

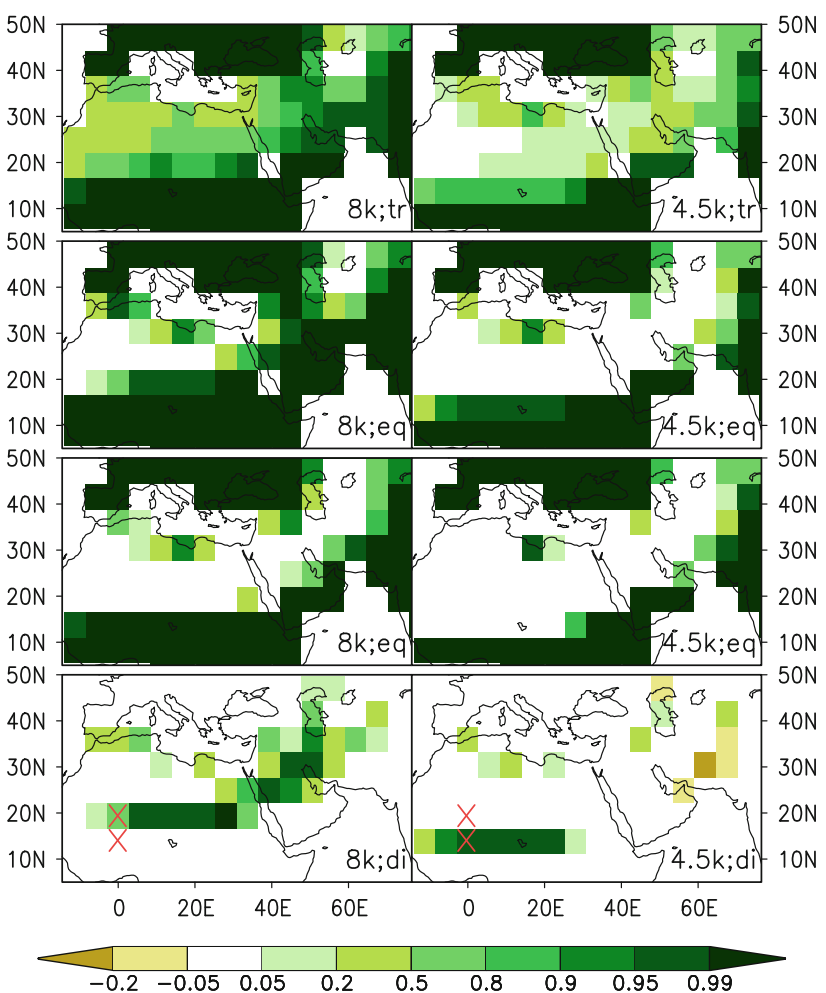

Fig. 6 Equilibrium vegetation cover fractions for transient (tr) and equilibrium (eq) mode with the modified VECODE. The second row of panels shows the steady state when starting from forest world conditions, the third row shows the steady state when starting from desert world conditions (all with 10 years coupling frequency). In the last row, the difference (di) between these states is displayed. Results for $8 \mathrm{k}$ are in the left column, results for $4.5 \mathrm{k}$ in the right column. The red crosses mark the two grid boxes used for Figs. 5 and 10

forest and desert run occurs at eight grid cells at the southern desert margin, now shifted to the south-west compared to $8 \mathrm{k}$. The green state in the bistable regions resembles the steady state solution of the transient mode. However, in the monostable desert regions the transient mode produces greener conditions than the equilibrium mode.

In short, multiple steady states occur only for the modified VECODE in equilibrium mode, but not between approx. $7 \mathrm{k}$ and $5 \mathrm{k}$ (Table 2). The latter is probably due to low resolution of our model and the concomitant large differences in precipitation between adjacent grid cells. As Fig. 1 illustrates, multiple steady states can only occur for a certain range of background precipitation $P_{0}$. Following this concept, background precipitation at the grid cell line around $20^{\circ} \mathrm{N}$ after $7 \mathrm{k}$ becomes small enough that the desert state remains the only equilibrium, while a green equilibrium is still the only possible state at the more southern grid cells around $14^{\circ} \mathrm{N}$. When background precipitation is further reduced, the bistability reappears at the southern grid 

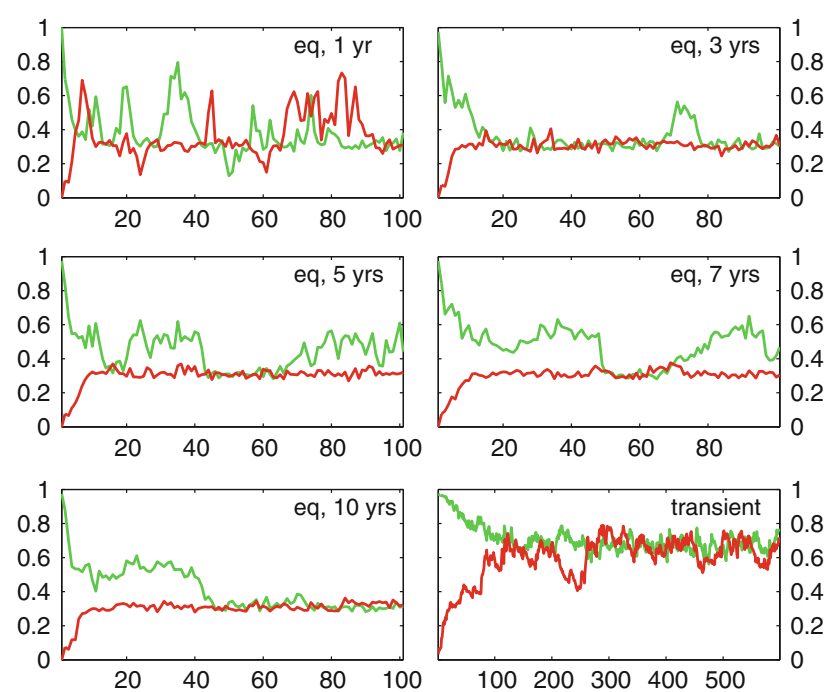

Fig. 7 Evolution of spatial mean vegetation cover in the Sahara/ Sahel region starting from forest (green) and desert world conditions $(r e d)$, in equilibrium mode (eq) with different coupling frequencies, as well as transient mode (transient). The $\mathrm{x}$-axis is in units of coupling iterations ( 1 year in case of transient coupling). All experiments have been performed with the modified VECODE version and under $8 \mathrm{k}$ conditions

cell line. In case of a higher resolution we would probably obtain a continuous shift of the bistable area to the southwest until its disappearance.

When comparing the geographical position of multiple states to the vegetation dynamics of our transient runs in Sect. 3, it becomes apparent that the most rapid vegetation decline occurs exactly in the bistable regions and shortly after the green state in equilibrium mode ceases to exist. In addition, the vegetation collapse is largest where the difference between the two states is high. Hence, the collapses in the transient experiments (in the Sahara, Arabia and the Middle East after 6k, and in the south-western Sahara after $4 \mathrm{k}$ ) can be interpreted as critical transitions resulting from a bifurcation in the atmosphere-vegetation system. However, the question remains why there is nonetheless no dependency on initial conditions in transient mode.

\subsubsection{Interpretation with the stochastic model}

The behaviour of a bistable system that exhibits variability is often exemplified with stochastic motion in a potential, whose two wells correspond to the system's basins of attraction (e.g. Fraedrich 1978, 1979; Wang and Eltahir 2000; Renssen et al. 2006; Scheffer et al. 2001, 2009; Ditlevsen and Johnsen 2010). If the noise level is small, the residence time in each well is very large and only one steady state can be observed in a time series. If the noise level is large compared to the potential barrier, a single peak is obtained in the probability density function (pdf) of the system's state variable at the potential's centre of gravity. In case of an intermediate noise level the system is supposed to flip irregularly from one regime to another, producing two separate peaks in the pdf. This behaviour is similar to PlaSim-VECODE in equilibrium mode: The more we reduce atmospheric variability by averaging over a larger number of years, the longer is the typical amount of time the system remains in the green regime (Fig. 7).

However, this concept is inconsistent with our PlaSimVECODE results in transient mode. The Lyapunov potential following Brovkin et al (1998) suggests two steady states for a certain range of $P_{0}$ :

$\frac{d V}{d t}=-\frac{d \Phi}{d V}=\frac{V^{*}\left(P^{*}(V)\right)-V}{\tau\left(P^{*}(V)\right)}$.

To obtain the potential $\Phi(V)$, Eq. 4 has to be integrated. As $\tau$ decreases with $V$, the green regime will be stabilised in comparison with the desert regime, and the change in stability can happen faster than for constant $\tau$. Nonetheless, the number and position of deterministic equilibria do not change. The above-mentioned inconsistency therefore remains: If the noise level is considered as small, the system's steady state would depend on initial conditions. If the noise level is intermediate, the stationary pdf should be bimodal. If the noise level is large, the mean state should lie between the deterministic equilibria, and the observed variability should be larger in amplitude than the distance between the deterministic equilibria. All this is not the case in our transiently coupled experiments (Table 2).

The reason is that the noise does not directly act on the changes in vegetation cover in Eq. 2 in the form of additive noise. Instead, a random precipitation anomaly in a particular year affects the vegetation change via the nonlinear relationship $V^{*}(P)$, on the basis of the interactive timescale $\tau(P)$. Therefore, the noise must be interpreted as multiplicative (Horsthemke and Lefever 1984).

We document these vital differences by calculating the empirical pdf from a time series of 10 million years from our stochastic model Eqs. 1, 2, and 3 (Fig. 8). We chose the equilibrium vegetation curve from the original VECODE, $P_{0}=60 \mathrm{~mm}$, and $k=300 \mathrm{~mm}$, so that the corresponding deterministic system has solutions at approx. $V=0$, and $V=0.7$. In the following, we always chose $G D D_{0}=10,000 \mathrm{~K}$ and $T=27^{\circ} \mathrm{C}$, as these are typical values in the Sahara/Sahel region in PlaSim-VECODE. The time series are calculated with FORTRAN-90, the pdfs with an advanced gaussian kernel density estimator for MATLAB (Botev et al. 2010). In order to always capture both possible equilibria, we start from $V=0$ and set $V$ to 1 after half the total time. The 50 years following each of these resets are not used for the analysis. In case a, we add Gaussian white noise directly to the dynamical equation (Eq. 2), while precipitation is set to its equilibrium value 

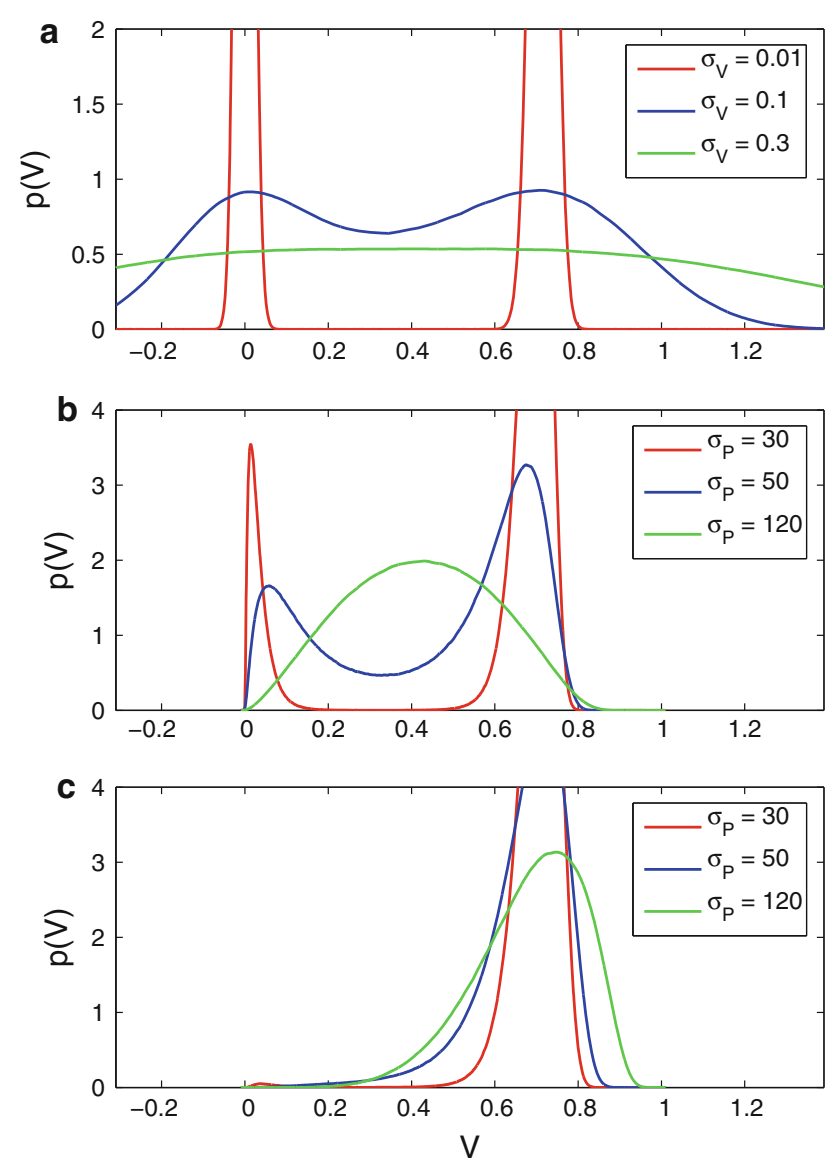

Fig. 8 Probability density functions of vegetation cover in the stochastic conceptual model with $P_{0}=60 \mathrm{~mm}$ and different noise levels. a Additive noise with $\tau=5$ years, $\mathbf{b}$ multiplicative noise with $\tau=5$ years, $\mathbf{c}$ multiplicative noise with interactive $\tau . \sigma_{P}$ is given in $\mathrm{mm} / \mathrm{year}$

$P^{*}(V)$ in each year, and $\tau$ is fixed at 5 years. For small noise levels, two peaks indicate the deterministic solutions, whereas for very large noise levels the bimodality disappears. In this additive case, we allow $V$ to be larger than 1 or smaller than 0 , for the sake of simplicity. In case $b$, we use Eq. 2 directly, treat precipitation as a Gaussian white noise process (while resetting negative precipitation values to 0 ), but still keep $\tau$ fixed at 5 years. This case corresponds to the stochastic model in Liu et al. (2006). Finally, in case $\mathrm{c}$ we also consider the dependency of precipitation on the vegetation timescale. The stochastic conceptual model is then fully described by Eq. 1, 2, and 3. For sufficiently large noise levels, the only steady state is again very similar to the deterministic green equilibrium, while the desert state has disappeared. The fact that the timescale of expansion is shorter than the timescale of vegetation dieback (Fig. 3) results in greener conditions in comparison to the case with a fixed timescale. The reason that no multiple steady states are found in transient mode is not simply the intensity of the noise itself, but rather the effect of the noise on the stability properties of the system. This effect corresponds to the noise-induced stability D'Odorico et al (2005) have found in their dryland vegetation model.

Atmospheric variability is also the reason why monostable desert regions tend to be greener in transient mode than in equilibrium mode: Although mean precipitation as such is not sufficient for vegetation, the critical threshold can be exceeded in exceptionally wet years and vegetation will temporarily establish. As vegetation cover cannot become negative, its temporal mean will thus also be positive. This effect corresponds to the greening effect described by Zeng et al. (2002). In equilibrium mode however, VECODE simulates a complete desert and the boundaries between desert and grass are more pronounced. Also in similarity to Zeng et al. (2002), these boundaries are more pronounced in the modified VECODE than in the original version, because the $V^{*}$ curve is then steeper.

\section{On the mechanism of vegetation collapse}

\subsection{Relation between system state and noise level}

It has been claimed that large atmospheric variability must lead to an only gradual decline in vegetation cover, for example as obtained in the intermediate complexity model of Renssen et al. (2003, 2006), and in the stochastic model of Liu et al (2006) in the case of white noise. Nonetheless, PlaSim-VECODE simulates an annual precipitation standard deviation of the order of $100 \mathrm{~mm}$, and can still exhibit a vegetation collapse. Therefore, the question arises why PlaSim-VECODE can exhibit rapid vegetation changes despite the large atmospheric variability.

To explain a similar behaviour in their model, Liu et al. (2006, 2007) suggested low frequency climate variability. Due to the memory effect of soil moisture, a small but sufficiently long lasting precipitation decline can cause a collapse of vegetation cover, even without a strong feedback between atmosphere and vegetation. In the uncoupled PlaSim we do not find any substantial autocorrelation of spatial mean annual precipitation in the Sahara/Sahel region (Fig. 9). Very similar results are obtained for each single grid cell. It becomes obvious that the decorrelation time of the atmosphere is considerably shorter than the timescale of grass in PlaSim-VECODE. Even when we calculate the autocorrelation function of soil moisture instead of precipitation, the results look very similar to Fig. 9. Hence, our assumption of white noise in the stochastic model is justified and the mechanism of collapse is not the same as in Liu et al. (2006, 2007).

Instead, it has to be considered that the noise level and the state of the system affect each other. In the dry desert with very low mean precipitation, the absolute variability 


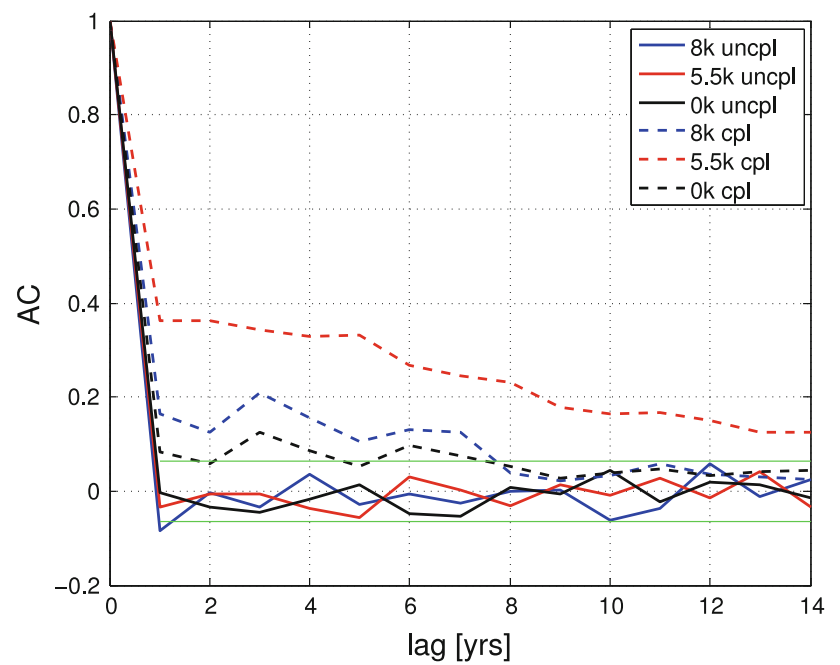

Fig. 9 Autocorrelation function of annual precipitation averaged over the Sahara/Sahel region for uncoupled (uncpl) and coupled (cpl) experiments in transient mode. From each run, the years 2,000-3,000 are used for the calculations. The $2 \sigma$-confidence bounds for white noise are indicated by the green lines

must also be small. For very low mean annual precipitation the distribution in PlaSim is similar to an exponential distribution but becomes more and more symmetric for increasing mean values. This property is obtained at every grid cell in the Sahara/Sahel region and is also independent of the orbit year. To capture these features as well as to avoid negative precipitation values we now represent precipitation as an inverse normal distribution in our stochastic model (Fig. 10). It is of further advantage that this distribution is described by the same parameters as the Gaussian distribution, mean and standard deviation. The mean $\mu_{P}$ still consists of the first two terms in Eq. 3. As the

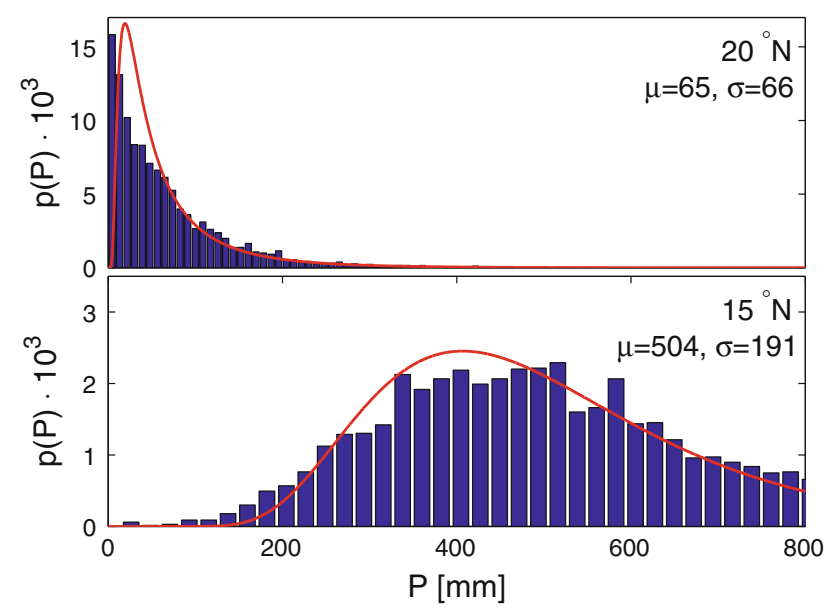

Fig. 10 Histograms of annual precipitation in the uncoupled PlaSim experiment TU-5.5k at the two grid cells marked in Fig. 6. Mean $\mu$ and standard deviation $\sigma$ of this data are given in $\mathrm{mm} / \mathrm{year}$. Each red line depicts the analytical inverse normal distribution that correspond to the two moments similarity between Fig. 11c and a, b illustrates, our results do not qualitatively depend on the choice of the distribution function.

To demonstrate the implications of the interaction between noise level and steady state we calculate the pdfs of vegetation cover fraction in our stochastic one-dimensional model for different values of background precipitation and two different noise levels $\sigma_{P}$ (Fig. 11a, b). The deterministic stability properties can be inferred from Fig. 1.

- For $P_{0}=70 \mathrm{~mm}$ and $P_{0}=60 \mathrm{~mm}$ two deterministic equilibria exist. The green state in this case is so stable that it is the only steady state for both noise levels. When by chance a dry event with only little vegetation occurs, and variability is reduced at the same time, the green state will only become more stable and the system returns to green conditions.
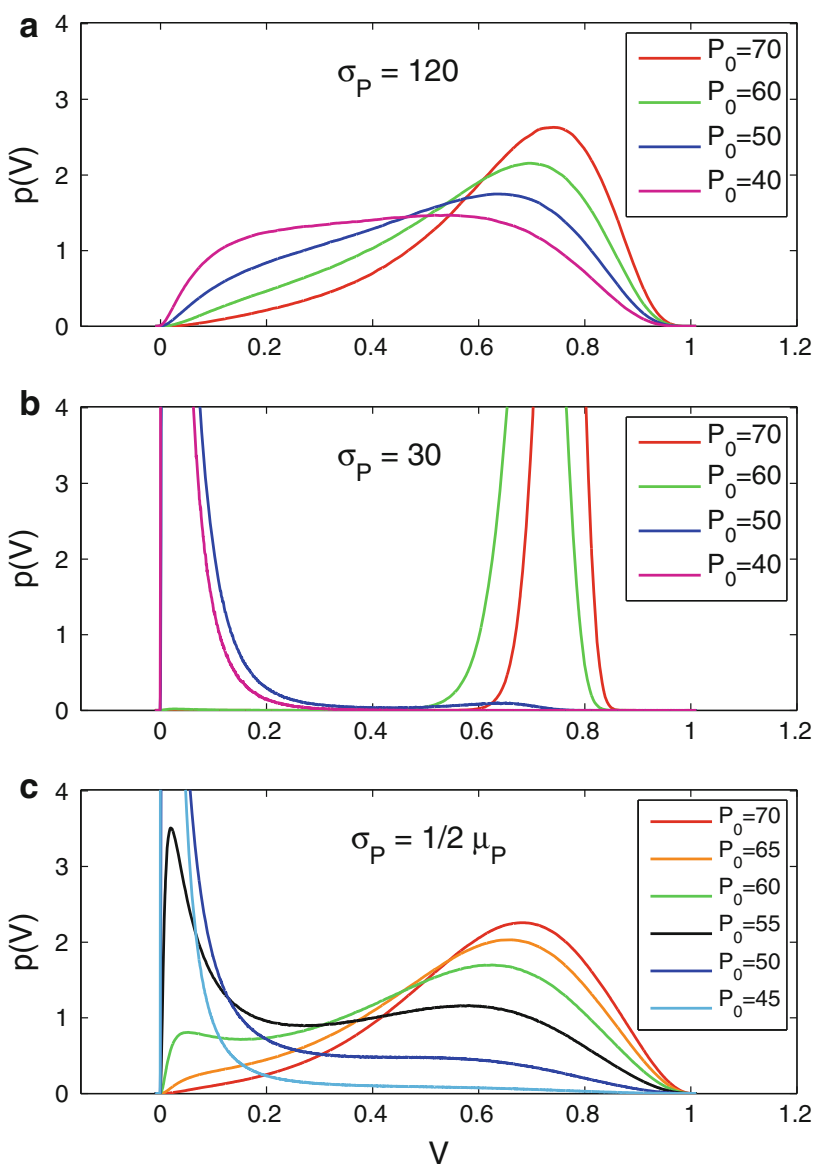

Fig. 11 Probability density functions of vegetation cover in the stochastic conceptual model for different values of $P_{0}$ at constant noise levels $\sigma_{P}(\mathbf{a}, \mathbf{b})$ and interactive noise level (c). Values of $P_{0}$ and $\sigma_{P}$ are in mm/year. The experiment length is $10^{7}$ years for fixed $\sigma_{P}$, and $10^{8}$ years for interactive $\sigma_{P} . \mu_{P}$ represents the mean of the precipitation probability distribution for a particular year 
- For $P_{0}=40 \mathrm{~mm}$, the desert state is the only deterministic equilibrium, although in the presence of large noise greener conditions occur as often as dry conditions.

- For approx. $P_{0}=50 \mathrm{~mm}$ the deterministic system is at a bifurcation point. The stability diagram then suggests a stable desert solution while the green equilibrium is only marginally stable. In the presence of large noise (Fig. 11a), green conditions still have maximum probability due to the noise. However, if one assumes that the noise level is reduced as soon as a state of low vegetation cover occurs, Fig. 11b applies. Suddenly, the desert state becomes more probable and the system may not escape from its dry state anymore.

To illustrate this concept we do not prescribe a fixed noise level anymore but calculate it from mean precipitation interactively, as suggested by our uncoupled experiments: For each single grid cell the relation between mean and standard deviation of annual precipitation is close to linear, especially under very dry conditions (Fig. 12). A similar relation holds in the transient experiments presented in Sect. 3, independent of the time period or grid cell. Therefore, a constant factor between mean and standard deviation of the precipitation distribution function, $r=\mu_{P} / \sigma_{P}$, seems justified, in analogy to D'Odorico et al. (2005). For the stochastic model we chose a value of $r=2$.

\subsection{The stochastic model with interactive noise level}

As a result of coupling the noise level to vegetation cover, the pdf will not change qualitatively in a large range of $P_{0}$, whereas it responds quite sensitively to $P_{0}$ around $55 \mathrm{~mm}$,

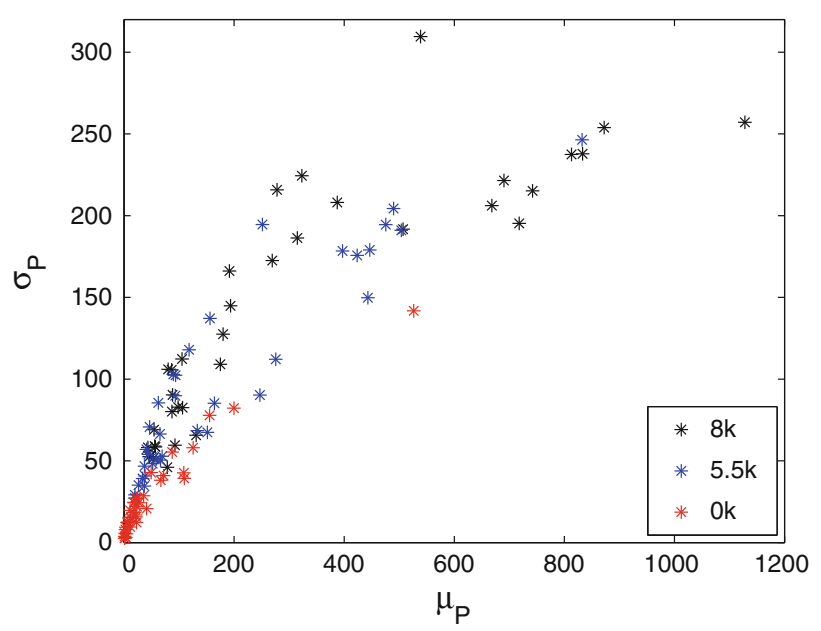

Fig. 12 Mean $\mu_{P}$ and standard deviation $\sigma_{P}$ of annual precipitation (both in $\mathrm{mm} /$ year) for all individual land grid cells of the Sahara/Sahel region in three uncoupled PlaSim experiments which is slightly larger than the critical point of the corresponding deterministic system. As the mean of the distribution rapidly shifts from high to low $V$ as $P_{0}$ falls below the critical threshold, the system can tip from a green state to a desert state, in analogy to a deterministic tipping point. However, in contrast to a deterministic catastrophic shift, no multiple states are involved: The stochastic model only shows a bimodal pdf in a parameter range that is much smaller than the hysteresis loop of its deterministic counterpart (approx. 50-60 mm, instead of 50-80 mm). Furthermore, the relative size of the peaks is exchanged almost abruptly as $P_{0}$ is varied (Fig. 11c).

It must be noted that the bimodal parameter range as well as the abruptness of a shift is much dependent on our choice of $r$. When analysing the pdfs of vegetation cover in PlaSim-VECODE (runs T-Orig-5.5k, as well as TF-Mod$4.5 \mathrm{k}$ or TD-Mod-4.5k), we also do not obtain any clear bimodality, neither for the spatial mean nor at any particular grid cell. In addition, no considerable hysteresis effect becomes apparent when comparing the experiments with forward (T-Mod-for1 and T-Mod-for2) and backward (TMod-back1 and T-Mod-back2) orbital forcing. For values of $r$ around 2, the conceptual stochastic model comes closest to these properties of PlaSim-VECODE. Hence, the conceptual model can explain the PlaSim-VECODE results, although we did not aim to derive precise parameter values for the conceptual stochastic model from PlaSim-VECODE. The agreement of $r=2$ to the results from individual grid cells (Fig. 12) is therefore convenient, but not compelling.

As in the deterministic case, the abruptness of the vegetation decline in our stochastic model also depends on the strength of the atmosphere-vegetation feedback. However, the system's behaviour during the transition period is subject to chance. To get an impression on the different possibilities we run the stochastic model approx. 50 times for $k=200 \mathrm{~mm}$ (weak feedback; monostable) and $k=300 \mathrm{~mm}$ (strong feedback; bistable between approx. $P_{0}=80 \mathrm{~mm}$ and $P_{0}=50 \mathrm{~mm}$ ). Figure 13 shows two realisations for each value of $k$. If the feedback is as strong as to allow for multiple equilibria, then sudden transitions always occur. Sometimes an early collapse is obtained, after which the system recovers before it finally drops into the desert state $(k=300 \mathrm{~mm}$, realisation 1$)$, a behaviour that resembles our experiment T-Mod-for1 (Fig. 5). In other (or often the very same) cases, a short post-collapse rebound occurs $(k=300 \mathrm{~mm}$, realisation 2$)$, a feature that also exists in some proxy records (e.g. deMenocal et al. 2000). In the case of the monostable system rapid changes can still occur $(k=200 \mathrm{~mm}$, realisation 1$)$, but usually the decline is more gradual as the unimodal pdf smoothly shifts towards lower $V(k=200 \mathrm{~mm}$, realisation 2). Considering the one-dimensional concept of the stability diagram, the 


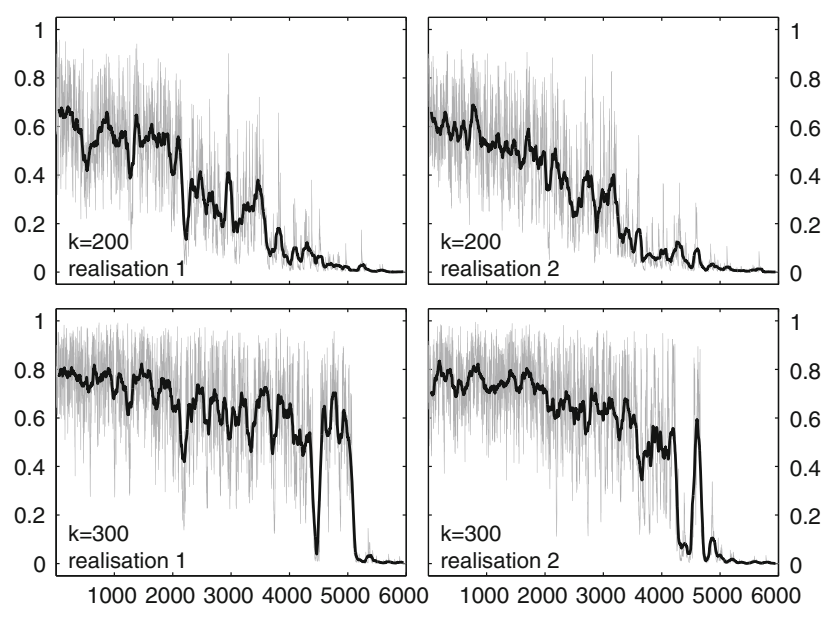

Fig. 13 Evolution of vegetation cover fractions in the stochastic conceptual model with interactive noise level as $P_{0}$ is reduced linearly over time from 120 to $30 \mathrm{~mm}$. For each feedback strength, weak ( $k=200 \mathrm{~mm}$, upper panels $)$ and strong $(k=300 \mathrm{~mm}$, lower panels $)$, two realisations have been selected

system must show an increased variance when the range of maximum slope in $V^{*}(P)$ is passed. This is due to the flattening of the corresponding Lyapunov potential (Brovkin et al. 1998), that causes slowing down of the system (Scheffer et al. 2009). The increased variability during the transition period in Renssen et al. (2003, 2006) can also be interpreted as such a temporary slowing down, instead of flickering between multiple states. In our stochastic model however, we generally do not obtain an increased variability in the weak feedback case, because the level of external noise is coupled to the state $V$ of the system which shows an overall decrease.

If we chose a particularly small feedback parameter (below $k=200 \mathrm{~mm}$ ), the stochastic model does not simulate any collapse. The stable collapse of Liu et al. (2006) (which is associated with an only gradual decline in precipitation) then remains the only mechanism to explain an abrupt vegetation decline.

\section{Summary and conclusions}

By performing experiments with PlaSim-VECODE for different ways of coupling, different strengths of the atmosphere-vegetation feedback, and different orbit years in the Holocene we have investigated the stability properties of PlaSim-VECODE and the nature of the transient evolution of vegetation cover in the Sahara/Sahel region. We have also illustrated our interpretation with a conceptual stochastic model.

Our results can be summarised as follows:

- A stability diagram is a good demonstration of the concept of multistability, but it must be applied with care when inferring the stability properties of a spatially heterogeneous model. In this case, it can show equilibria that do not exist.

- The detection of multiple equilibria by choosing different initial conditions depends on the method of coupling between atmosphere and vegetation model. Atmospheric variability can obliterate a deterministic bistability, but beyond that it can act as multiplicative noise and change the stability properties themselves.

- A vegetation collapse is possible despite large and uncorrelated climate variability, provided 1 . a positive and sufficiently strong atmosphere-vegetation feedback, and 2. an impact of the system's state on the intensity of variability. PlaSim-VECODE is an example for such a mechanism: Before the vegetation collapse, the green state is stabilised by the climate-dependent timescale and the large atmospheric variability. Once the system comes close to the desert state and background precipitation is sufficiently low, the desert state is stabilised due to the decrease in variability.

Our results imply that climate variability, in interaction with nonlinearities in the climate system, as well as spatial complexity must be considered for stability analysis. It also becomes apparent that the exact evolution of the system (such as abruptness of the transition, or the timing and existence of a post-collapse rebound) can strongly depend on the realisation. Even a most realistic climate model can then not be expected to agree with observations.

It also remains unclear which mechanism most appropriately describes the Saharan vegetation decline in reality. This question cannot be answered on the basis of our model results alone. Limitations arise from the low resolution and the simplicity compared to comprehensive Earth System Models. Specifically, VECODE is an empirical rather than a process-based model, and it is lacking an annual cycle. Although soil moisture is an essential quantity of the atmosphere-vegetation system (Wang and Eltahir 2000; D'Odorico et al. 2005), it is not represented in the model. However, as annual soil moisture in PlaSim also shows a white noise spectrum, a different coupling would probably not affect our results. However, our way of calculating surface parameters and evapotranspiration is very crude. Also, it is certainly not realistic to tie all physical surface properties that depend on vegetation cover to the same timescale.

The treatment of this timescale $\tau$ is a critical component in VECODE because of its influence on the steady state. The relation $\tau(P)$ originates from global observations of biomass turnover time in different ecosystems (Brovkin et al. 1997). According to these observations, dry ecosystems are dominated by woody plant types such as shrubs, which have a slower turnover rate than grasses in wet 
ecosystems. It is thus not compelling that the same relation should also hold for fast precipitation changes at a particular location. The model's response to large atmospheric variability may therefore not be appropriate. For example, a sudden interruption of all precipitation would result in an unrealistically slow dieback as between $4 \mathrm{k}$ and $2 \mathrm{k}$ in Fig. 5 (top panels). On the other hand, plant establishment and dieback in reality are due to very different biological processes and plant traits are known to adapt to changes in climate. Assuming a constant timescale as in previous conceptual studies may therefore also not be realistic. In this regard, our study tests the implications of a climate dependent timescale for a model's stability properties such as sensitivity to initial conditions. Furthermore, our main conclusions as summarised above do not rely on the exact formulation of $\tau(P)$. In particular, a variable timescale is not an essential prerequisite for a collapse of a system that is subject to white noise. The reduction of variability after a shift has occurred can already be sufficient for a sudden transition, a feature that certainly applies to dry deserts.

Concerning climate variability the lack of ocean dynamics is another limitation in our model studies, as the ocean introduces more variability, especially at low frequencies. Including the ocean would also influence the stability properties of the model (Zeng and Neelin 2000), for example, the SST-monsoon-feedback could enhance the effect of the atmosphere-vegetation feedback (Liu et al. 2003, 2004).

Apart from these uncertainties with regard to the real climate system, the question arises how applicable our results are to other models. It is self-evident that conceptual vegetation models with constant stochastic forcing and a fixed timescale can generally be descibed by the conventional approach of stochastic motion in a deterministic potential. The stability properties of the system may then be inferred from time series or even from the model formulation. However, both is not the case for models of high complexity. Whether our findings apply to any particular complex vegetation model remains an open question, because such models describe many different processes on different timescales, while there is essentially only one variable and one timescale determining grass cover in VECODE. As the atmosphere model's sensitivity to land cover changes also affects the strength of the atmospherevegetation feedback, stability properties are not determined by the vegetation model alone. In addition, the behaviour of the coupled model depends on the magnitude and the spectral properties of atmospheric variability. In the light of these aspects it is suggestive that properties similar to PlaSim-VECODE may also occur in other models.

Therefore, general implications for the detection of multistability and tipping points in climate models become apparent. Claussen (2008) summarises different methods that have been followed to identify multistable regions or subsystems in the earth system ("hot spots"): the choice of different initial conditions, the construction of a stability diagram, the analysis of variability under stationary conditions, the application of so-called "early warning signals" for a system that approaches a bifurcation point (Scheffer et al. 2009), and the identification and quantification of mechanisms that can cause runaway feedbacks. Our results indicate that most of these methods may not be reliable under all circumstances: While variability can impede the first, and spatial complexity the second strategy, the stationary variability may also not provide the expected clues about the stability of a system. PlaSim-VECODE is a good example: Before the vegetation collapse in experiments T-Mod-for1 and T-Mod-for2, the system can reach a state similar to post-collapse conditions at some times. However, the pdf remains unimodal. With regard to these results it is suggestive that the applicability of early warning signals should be further studied, and that a good understanding of the underlying processes may be indispensable to detect and predict tipping points.

Acknowledgments We are grateful for the constructive comments of our anonymous reviewers. Also, we would like to thank Zhengyu Liu, Victor Brovkin and Christian Reick for the detailed and helpful discussions. Dr Sheldon Cooper is gratefully acknowledged for his singular remarks. KF, Max Planck Fellow, acknowledges the support by the Max Planck Society. This work is funded by Deutsche Forschungsgesellschaft, Cluster of Excellence 'CliSAP' (DFG EXC 177). The simulations were performed at the German Climate Computing Center (DKRZ). The service charges for this publication have been covered by the Max-Planck-Society.

Open Access This article is distributed under the terms of the Creative Commons Attribution Noncommercial License which permits any noncommercial use, distribution, and reproduction in any medium, provided the original author(s) and source are credited.

\section{References}

Botev ZI, Grotowski JF, Kroese DP (2010) Kernel density estimation via diffusion. Ann Stat 38(5):2916-2957

Braconnot P, Otto-Bliesner B, Harrison S, Joussaume S, Peterchmitt JY, Abe-Ouchi A, Crucifix M, Driesschaert E, Fichefet T, Hewitt CD, Kageyama M, Kitoh A, Laine A, Loutre MF, Marti O, Merkel U, Ramstein G, Valdes P, Weber SL, Yu Y, Zhao Y (2007) Results of PMIP2 coupled simulations of the midHolocene and last glacial maximum-part 1: experiments and large-scale features. Clim Past 3(2):261-277

Brovkin V, Ganopolski A, Svirezhev Y (1997) A continuous climatevegetation classification for use in climate-biosphere studies. Ecol Model 101(2-3):251-261

Brovkin V, Claussen M, Petoukhov V, Ganopolski A (1998) On the stability of the atmosphere-vegetation system in the Sahara/ Sahel region. J Geophys Res Atmos 103(D24):31613-31624

Brovkin V, Bendtsen J, Claussen M, Ganopolski A, Kubatzki C, Petoukhov V, Andreev A (2002) Carbon cycle, vegetation, and 
climate dynamics in the Holocene: experiments with the CLIMBER-2 Model. Global Biogeochem Cy 16(4):1139. doi: 10.1029/2001GB001662

Brovkin V, Levis S, Loutre MF, Crucifix M, Claussen M, Ganopolski A, Kubatzki C, Petoukhov V (2003) Stability analysis of the climate-vegetation system in the northern high latitudes. Climatic Change 57(1-2):119-138

Charney JG (1975) Dynamics of deserts and drought in the Sahel. Q J Roy Meteor Soc 101(428):193-202

Claussen M (1994) On coupling global biome models with climate models. Climate Res 4(3):203-221

Claussen M (1997) Modeling bio-geophysical feedback in the African and Indian monsoon region. Clim Dynam 13(4):247-257

Claussen M (1998) On multiple solutions of the atmospherevegetation system in present-day climate. Global Change Biol 4(5):549-559

Claussen M (2008) Holocene rapid land cover change-evidence and theory. In: Battarbee R, Binney $\mathrm{H}$ (eds) Natural climate variability and global warming. Blackwell Publishing, Oxford, pp 232-253

Claussen M (2009) Late Quaternary vegetation-climate feedbacks. Clim Past 5(2):203-216

Claussen M, Kubatzki C, Brovkin V, Ganopolski A, Hoelzmann P, Pachur HJ (1999) Simulation of an abrupt change in Saharan vegetation in the mid-Holocene. Geophys Res Lett 26(14): 2037-2040

Dekker SC, de Boer HJ, Brovkin V, Fraedrich K, Wassen MJ, Rietkerk M (2010) Biogeophysical feedbacks trigger shifts in the modelled vegetation-atmosphere system at multiple scales. Biogeosciences 7(4):1237-1245

deMenocal P, Ortiz J, Guilderson T, Adkins J, Sarnthein M, Baker L, Yarusinsky M (2000) Abrupt onset and termination of the African Humid Period: rapid climate responses to gradual insolation forcing. Quaternary Sci Rev 19(1-5):347-361

Ditlevsen PD, Johnsen SJ (2010) Tipping points: early warning and wishful thinking. Geophys Res Lett 37:L19,703. doi:10.1029/ 2010GL044486

D'Odorico P, Laio F, Ridolfi L (2005) Noise-induced stability in dryland plant ecosystems. P Natl Acad Sci USA 102(31): 10819-10822. doi:10.1073/pnas.0502884102

Fraedrich K (1978) Structural and stochastic analysis of a zerodimensional climate system. Q J Roy Meteor Soc 104(440): $461-474$

Fraedrich K (1979) Catastrophes and resilience of a zero-dimensional climate system with ice-albedo and greenhouse feedback. Q J Roy Meteor Soc 105(443):147-167

Fraedrich K, Kleidon A, Lunkeit F (1999) A green planet versus a desert world: estimating the effect of vegetation extremes on the atmosphere. J Climate 12(10):3156-3163

Fraedrich K, Jansen H, Kirk E, Luksch U, Lunkeit F (2005) The Planet Simulator: towards a user friendly model. Meteorol Z 14(3):299-304. doi:10.1127/0941-2948/2005/0043

Fraedrich K, Jansen H, Kirk E, Lunkeit F (2005) The Planet Simulator: green planet and desert world. Meteorol Z 14(3):305314. doi:10.1127/0941-2948/2005/0044

Guttal V, Jayaprakash C (2007) Impact of noise on bistable ecological systems. Ecol Model 201(3-4):420-428. doi:10.1016/j. ecolmodel.2006.10.005

Hales K, Neelin JD, Zeng N (2004) Sensitivity of tropical land climate to leaf area index: role of surface conductance versus albedo. J Climate 17(7):1459-1473

Horsthemke W, Lefever R (1984) Noise-induced transitions. Springer, New York

Irizarry-Ortiz MM, Wang GL, Eltahir EAB (2003) Role of the biosphere in the mid-Holocene climate of West Africa. J Geophys Res Atmos 108(D2)
Kroepelin S, Verschuren D, Lezine AM, Eggermont H, Cocquyt C, Francus P, Cazet JP, Fagot M, Rumes B, Russell JM, Darius F, Conley DJ, Schuster M, von Suchodoletz H, Engstrom DR (2008) Climate-driven ecosystem succession in the Sahara: the past 6,000 years. Science (Washington, DC) 320(5877):765-768

Levis S, Foley JA, Brovkin V, Pollard D (1999) On the stability of the high-latitude climate-vegetation system in a coupled atmosphere-biosphere model. Global Ecol Biogeogr 8(6):489-500

Lezine AM (2009) Timing of vegetation changes at the end of the Holocene Humid Period in desert areas at the northern edge of the Atlantic and Indian monsoon systems. Compt Rendus Geosci 341(8-9):750-759

Liu Z, Gallimore RG, Kutzbach JE, Xu W, Golubev Y, Behling P, Selin R (1999) Modeling long-term climate changes with equilibrium asynchronous coupling. Clim Dynam 15(5):325-340

Liu Z, Otto-Bliesner B, Kutzbach J, Li L, Shields C (2003) Coupled climate simulation of the evolution of global monsoons in the Holocene. J Climate 16(15):2472-2490

Liu Z, Harrison SP, Kutzbach J, Otto-Bliesner B (2004) Global monsoons in the mid-Holocene and oceanic feedback. Clim Dynam 22(2-3):157-182

Liu Z, Wang Y, Gallimore R, Gasse F, Johnson T, deMenocal P, Adkins J, Notaro M, Prenticer IC, Kutzbach J, Jacob R, Behling P, Wang L, Ong E (2007) Simulating the transient evolution and abrupt change of Northern Africa atmosphere-ocean-terrestrial ecosystem in the Holocene. Quaternary Sci Rev 26(13-14): 1818-1837. doi:10.1016/j.quascirev.2007.03.002

Liu ZY (2010) Bimodality in a monostable climate-ecosystem: the role of climate variability and soil moisture memory. J Climate 23(6): 1447-1455

Liu ZY, Wang Y, Gallimore R, Notaro M, Prentice IC (2006) On the cause of abrupt vegetation collapse in North Africa during the Holocene: climate variability vs. vegetation feedback. Geophys Res Lett 33(22). doi:10.1029/2006GL028062

Livina VN, Kwasniok F, Lenton TM (2010) Potential analysis reveals changing number of climate states during the last $60 \mathrm{kyr}$. Clim Past 6(1):77-82

Oyama MD, Nobre CA (2003) A new climate-vegetation equilibrium state for tropical South America. Geophys Res Lett 30(23): CLM5-1-CLM5-4

Pinty B, Roveda F, Verstraete MM, Gobron N, Govaerts Y, Martonchik JV, Diner DJ, Kahn RA (2000) Surface albedo retrieval from Meteosat-2. Applications. J Geophys Res Atmos 105(D14): 18,113-18,134

Prentice IC, Jolly D, participants B (2000) Mid-Holocene and glacialmaximum vegetation geography of the northern continents and Africa. J Biogeogr 27(3):507-519

Renssen H, Brovkin V, Fichefet T, Goosse H (2003) Holocene climate instability during the termination of the African Humid Period. Geophys Res Lett 30(4):1184. doi:10.1029/2002 GL016636

Renssen H, Brovkin V, Fichefet T, Goosse H (2006) Simulation of the holocene climate evolution in northern africa: the termination of the African Humid Period. Quat Int 150:95-102

Salzmann U, Hoelzmann P (2005) The Dahomey Gap: an abrupt climatically induced rain forest fragmentation in West Africa during the late Holocene. Holocene 15(2):190-199

Scheffer M, Carpenter S, Foley JA, Folke C, Walker B (2001) Catastrophic shifts in ecosystems. Nat Biotechnol 413(6856): 591-596

Scheffer M, Bascompte J, Brock WA, Brovkin V, Carpenter SR, Dakos V, Held H, van Nes EH, Rietkerk M, Sugihara G (2009) Early-warning signals for critical transitions. Nat Biotechnol 461(7260):53-59. doi:10.1038/nature08227

Schurgers G, Mikolajewicz U, Groger M, Maier-Reimer E, Vizcaino M, Winguth A (2006) Dynamics of the terrestrial biosphere, 
climate and atmospheric $\mathrm{CO}_{2}$ concentration during interglacials: a comparison between Eemian and Holocene. Clim Past 2(2):205-220

Stull R (1988) An introduction to boundary layer meteorology. Reidel Publishing Co, Dordrecht

Timmermann A, Lohmann G (2000) Noise-induced transitions in a simplified model of the thermohaline circulation. J Phys Oceanogr 30(8):1891-1900

Vincens A, Buchet G, Servant M, Collaborators EM (2010) Vegetation response to the 'African Humid Period' termination in Central Cameroon ( 7 degrees $\mathrm{N}$ ) - new pollen insight from Lake Mbalang. Clim Past 6(3):281-294
Wang GL (2004) A conceptual modeling study on biosphereatmosphere interactions and its implications for physically based climate modeling. J Climate 17(13):2572-2583

Wang GL, Eltahir EAB (2000) Biosphere-atmosphere interactions over West Africa. II: multiple climate equilibria. Q J Roy Meteor Soc 126(565): 1261-1280

Zeng N, Neelin JD (2000) The role of vegetation-climate interaction and interannual variability in shaping the African savanna. J Climate 13(15):2665-2670

Zeng N, Hales K, Neelin JD (2002) Nonlinear dynamics in a coupled vegetation-atmosphere system and implications for desert-forest gradient. J Climate 15(23):3474-3487 